
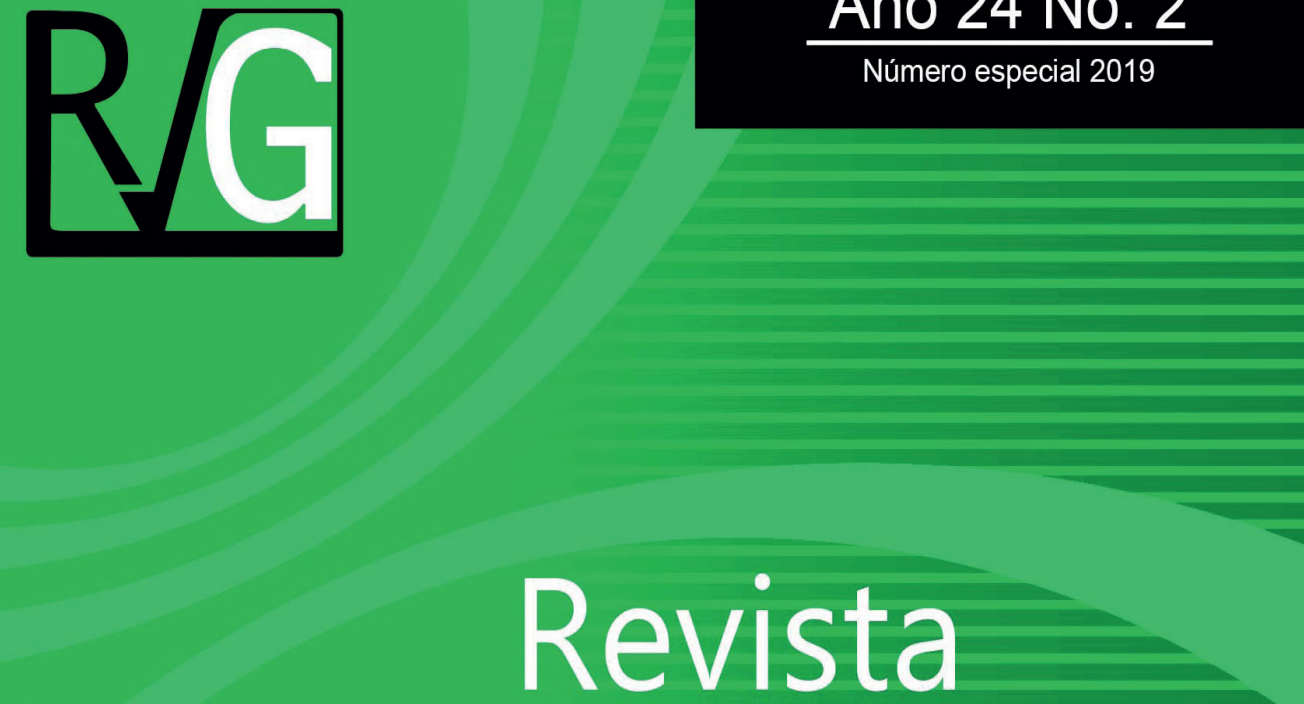

Venezolana de

$\frac{1}{6}$

$\frac{\mathbb{O}}{\frac{1}{0}}$
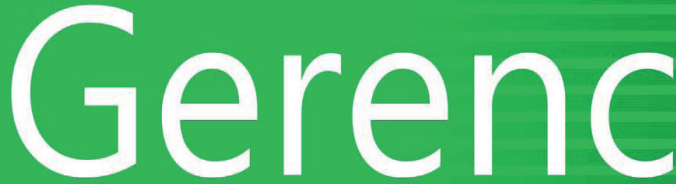

0

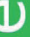

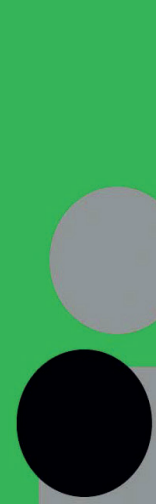

ro

u

$>$

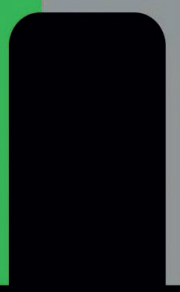




\title{
Prácticas ambientales en empresas manufactureras de equipo de transporte en México'
}

\author{
González Acolt, Roberto² \\ Castillo Rodríguez, Jesús ${ }^{3}$ \\ Ivanova Boncheva, Antonina ${ }^{4}$
}

\section{Resumen}

Las empresas del subsector equipo de transporte (automotriz, aeroespacial y otras unidades de transporte) en México generan un número importante de empleos y sobresalen por su dinamismo tecnológico. No obstante, poco se conoce sobre sus prácticas a favor del medio ambiente. Este trabajo tuvo como objetivo estudiar las prácticas de protección y mejora del medio ambiente de este tipo de empresas. La

Recibido: 11-09-19 Aceptado: 30-10-19

1 El artículo forma parte del proyecto del Programa de Investigaciones Económicas Administrativas (PIEA) 17-1 del sistema de proyectos de investigación de la Dirección General de Investigación y Posgrado de la Universidad Autónoma de Aguascalientes (UAA). Se agradece el apoyo financiero otorgado por la UAA.

2 Doctor en Ciencias con Orientación en Economía. Profesor-Investigador del departamento de Economía de la Universidad Autónoma de Aguascalientes. Responsable del Cuerpo Académico Desarrollo Local. E-mail: rgonza@correo.uaa.mx, ORCID: https://orcid.org/0000-0001-54683028

3 Estudiante del Doctorado en Ciencias Administrativas de la Universidad Autónoma de Aguascalientes. E-mail: cast86@prodigy.net.mx, ORCID: https://orcid.org/0000-0003-1497-9451

4 Doctora en Economía. Profesora Investigadora tiempo completo del departamento de Economía de la Universidad Autónoma de Baja California Sur. E-mail:aivanova@uabcs.mx, http:// orcid.org/0000-0003-1591-6006 
metodología consistió en analizar cualitativa y cuantitativamente las respuestas que dieron a un cuestionario sobre medio ambiente una muestra de 1047 establecimientos del subsector equipo de transporte. Los resultados indican que en relación al número de empresas, es significativo las que separan residuos y desechos; muy bajo las que invierten y gastan en disminuir la contaminación al aire y agua; un poco más de la mitad cumple con alguna norma de carácter ambiental; relativamente, bajo las que reciclan, aplican algún tratamiento a las aguas residuales o cuentan con personal dedicado a labores de protección del medio ambiente y los recursos naturales. Se concluye que la relevancia económica de las empresas de este subsector no se ve refleja en la aplicación generalizada de prácticas de protección y mejora del medio ambiente

Palabras clave: prácticas ambientales; subsector equipo de transporte; medio ambiente; empresas manufactureras: México.

\title{
Environmental practices in manufacturing companies of transportation equipment in Mexico
}

\begin{abstract}
The firms of the transport equipment subsector (industry automotive, aerospace and other transport companies) in Mexico generate a significant number of jobs and stand out for their technological dynamism. However, little is known about their practices in favor of the environment. This paper examines the practices of protection and improvement of the environment of this type of firms. The methodology consisted of qualitatively and quantitatively analyzing the responses that gave a questionnaire on the environment a sample of 1047 firms of the transportation equipment subsector. Our results shows that in relation to the number of companies, it is significant those that separate waste and scrap; very few those that invest and spend to reduce air and water pollution; a little more than half complies with some environmental norm; relatively, under which they recycle, apply some treatment to wastewater or have personnel dedicated to environmental protection and natural resources. It is concluded that the economic relevance of the firms in this subsector is not reflected in the widespread application of environmental protection and improvement practices.
\end{abstract}

Keywords: environmental practices; subsector transport equipment; environment; Mexico. 


\section{Introducción}

El medio ambiente como un elemento de competitividad constituye en determinadas empresas uno de los factores que explica sus decisiones de gestión y su manejo en la reducción de los impactos negativos que originan sobre el medio ambiente y los recursos naturales. Este comportamiento, lamentablemente, no está generalizado al conjunto de todas las empresas. Azqueta et al, (2007:330) describen una clasificación de las empresas de acuerdo a la actitud que asumen ante la problemática ambiental, la tipología abarca aquellas con una percepción negativa que juzgan como distorsionantes las medidas de mejoramiento del medio ambiente dado que dificulta la obtención de beneficios; le siguen las pasivas indiferentes que se caracterizan por atender de manera esporádica y obligatoria los problemas ambientales que producen; en tercer lugar aparecen las reactivas que responden con acciones a favor del medio ambiente con el único objetivo de cumplir con la normatividad ambiental; en cuarto sitio se tiene las proactivas que incorporan las variables ambientales en sus sistemas de gestión con la finalidad de anticiparse a determinadas regulaciones o para obtener una mayor competitividad en el mercado; por último, las líderes que asumen un compromiso social favorable al medio ambiente $y$ consideran que éste es una oportunidad para mantener su consolidación en el mercado.

Obsérvese que este comportamiento ante el medio ambiente de las empresas va desde aquellas que mantienen una posición de resistencia y pasividad hasta las que sostienen una perspectiva positiva y de compromiso social en la que la búsqueda de un mayor beneficio económico que no se contrapone con la implementación de acciones a favor del medio ambiente. Ahora bien, este comportamiento ambiental de las firmas es influido por un conjunto de factores entre los cuales se puede mencionar (Earnhart et al, 2014:166): los Recursos y capacidades de la empresa.

En este enfoque teórico, se establece que el capital físico y humano, la tecnología y la cultura corporativa son parte esencial de la estrategia corporativa de las empresas. La elección que realice la empresa de estos factores constituye en el largo plazo una ventaja comparativa para la empresa. Bajo este enfoque la maximización de la ganancia de las empresas implica diseñar estrategias productivas de bajo costo y favorables hacia un medio ambiente más limpio.

Las presiones del mercado y la competencia obliga a las empresas a tomaraccionesqueimpliquendisminución de costos en el tratamiento y uso de los insumos productivos. Asimismo, las firmas buscan ampliar su presencia en el mercado, atrayendo a consumidores cada vez más preocupados por adquirir bienes y servicios que no impliquen grandes daños al medio ambiente, o a inversionistas con inquietud por financiar a empresas con una filosofía de sustentabilidad.

En lo que respecta a las regulaciones gubernamentales, el gobierno mediante un conjunto de instrumentos de política pública puede afectar el comportamiento ambiental de las empresas. Dentro del conjunto de medidas sobre el medio ambiente que el gobierno puede aplicar se tiene: políticas de mandato y control (leyes, reglamentos, estándares), políticas de incentivos (impuestos, subsidios, 
permisos de emisión comerciables) o políticas de programas voluntarios (auditorias ambientales).

De igual forma, la presión de la sociedad civil, con diferentes integrantes conformados en Organizaciones no Gubernamentales, comolasasociaciones ambientalistas, cada vez tienen mayor presencia y voz en la búsqueda de la conservación y buen uso de los recursos naturales y en la disminución de la contaminación ambiental que producen los diferentes agentes económicos. Mientras mayor peso e injerencia tengan estos grupos en la opinión pública mayor presión habrá para que en los aparatos gubernamentales o en las empresas se tomen decisiones de mejora del medio ambiente.

De cierta manera, la actividad económica donde se localice la unidad productiva es un aspecto que esta presente en su respuesta ante la problemática ambiental, por ejemplo, es más probable que las empresas manufactureras, debido a que sus procesos productivos implican contaminación aguda en el aire y agua apliquen medidas de reducción o eliminación de esta forma de contaminación.

En México, existen ramas dentro del sector manufacturero que originan un elevado grado de contaminación como es el caso de la petroquímica, química, papel, petróleo, metálicas básicas, entre otras (Romo et al, 2005:134). De hecho, este sector es una de las actividades económicas con mayores costos por agotamiento de los recursos naturales y degradación del medio ambiente ${ }^{6}$.

Existen estudios interesantes que analizan las practicas ambientales de algunas ramas del sector manufacturero en México, por ejemplo, Brown (2008) analizó si el grado de apertura comercial implicó que la industria textil mexicana tuviera un mejor desempeño ambiental. A su vez, Medina (2008) estudió los determinantes y los obstáculos en la adopción de esquemas voluntarios de gestión ambiental en la industria química de México. Muller y Kolk (2009) examinan la Responsabilidad Social Corporativa (RSC) en 93 empresas de autopartes mexicanas, la RSC la miden bajo la dimensión ambiental, laboral y comunitaria, los autores encontraron, con respecto a la dimensión ambiental de la RSC, que el uso de las fuentes de energía renovables dentro del total de la energía consumida es bajo en la mayoría de las firmas (aproximadamente 83.1), no obstante éstas mantienen un nivel de reciclaje de residuos relativamente alto y más del $75 \%$ de las compañias encuestadas al menos dedica más de la mitad de un día al entrenamiento ambiental de sus trabajadores.

Los estudios citados contibuyen a comprender la compleja relación de la aplicación de medidas de mejora del medio ambiente de industrias especificas manufactureras en México, sin embargo, es necesario seguir ampliando este análisis a otras actividades industriales

6 Los costos por agotamiento de los recursos naturales y degradación del medio ambiente son aquellos en los que incurriría la sociedad para remediar, restituir o prevenir el agotamiento y degradación de los recursos naturales y el medio ambiente, producto de las actividades económicas (INEGI, 2012:156). 
como es el caso del subsector de equipo de transporte ${ }^{7}$. En México, las ramas de este subsector como la industria automotriz tienen mucha importancia en cuanto a la generación de empleos y su integración con otras actividades productivas, también, aunque en años mas recientes, la industria aeroespacial se ha posesionado como una rama relevante por su sustento tecnológico y las posibilidades que ofrece para que otras empresas mexicanas se incorporen a su sistema de proveeduría.

Unger (2010) en su trabajo sobre los clusters industriales en México, cataloga a la industria aeroespacial como una actividad productiva de bienes modernos con un elevado dinamismo tecnológico, mientras que la industria automotriz la clasifica como productora de bienes tecnológicamente maduros e intensivas en escala. Para Unger (2010) el espacio regional -donde se conjugan la disponibilidad de recursos naturales, los sistemas de innovación, las redes industriales y otros factores juegan un papel central en el desarrollo y consolidación de estos clusters. La industria automotriz -con relación a la aeronáutica- lleva más tiempo establecida en México y es en la década de los noventa cuando detona su expansión hasta alcanzar su consolidación actual (Romero, 2011), mientras que la actividad de la aeronáutica inició su crecimiento en la década del 2000 y está en proceso de desarrollo (Hernández, 2015). Existen investigaciones interesantes vinculados a estas ramas productivas, por ejemplo sin embargo, con base a la literatura consultada, son escasos los estudios que se enfocan a comprender las prácticas de protección o mejora del medio ambiente de estas actividades productivas, por lo que este trabajo pretende contribuir a la comprensión de este fenómeno.

La metodología se sustentó en un análisis cualitativo y cuantitativo de las respuestas de 1047 empresas del subsector equipo de transporte a un cuestionario sobre medio ambiente, desarrollado por INEGI (2014). La información de la encuesta, particularmente, se relacionó con las siguientes prácticas de protección y mejora del medio ambiente de las empresas del subsector referido: a) gastos realizados en bienes $y$ servicios o inversión en maquinaria, equipo e instalaciones para disminuir o eliminar la contaminación del aire o los contaminantes en aguas residuales; b) empleados destinados a actividades de protección del medio ambiente o de los recursos naturales; c) cumplimiento con alguna norma vinculada al medio ambiente; d) utilización de materiales reciclados: materias primas o materiales de empaque y embalaje; separación de residuos o desechos generados; tratamiento de aguas residuales

Por otra parte, para un mejor estudio de las prácticas ambientales de estas empresas, se agrutinó las ramas industriales que integran al subsector en tres grupos de la siguiente forma: 1) industria automotriz -incluyó fabricación de automóviles, camionetas y autobuses; carrocerías y remolques; partes para vehículos y automotores-; 2)

7 Este subsector esta integrado principalmente por la industria automotriz (fabricación de automóviles, camiones, carrocerias, remolques, partes para vehículos), aeroespacial y otros equipos de transporte (INEGI, 2013) 
industria aeroespacial; 3) otros equipos de transporte - abarcó equipo ferroviario, embarcaciones y otros-.

\section{Protección y mejora del medio ambiente en empresas manufactureras}

En el gráfico 1 se aprecia que la baja participación de las empresas del subsector fabricación de equipo de transporte que efectuan gasto corriente (materiales y servicios) o de inversión (instalaciones o equipo) para disminuir las emisiones al aire, siendo las empresas aeroespaciales las que menos realizan este tipo de gasto.

\section{Gráfico 1}

Gasto corriente o de inversión para disminuir las emisiones al aire (\%)

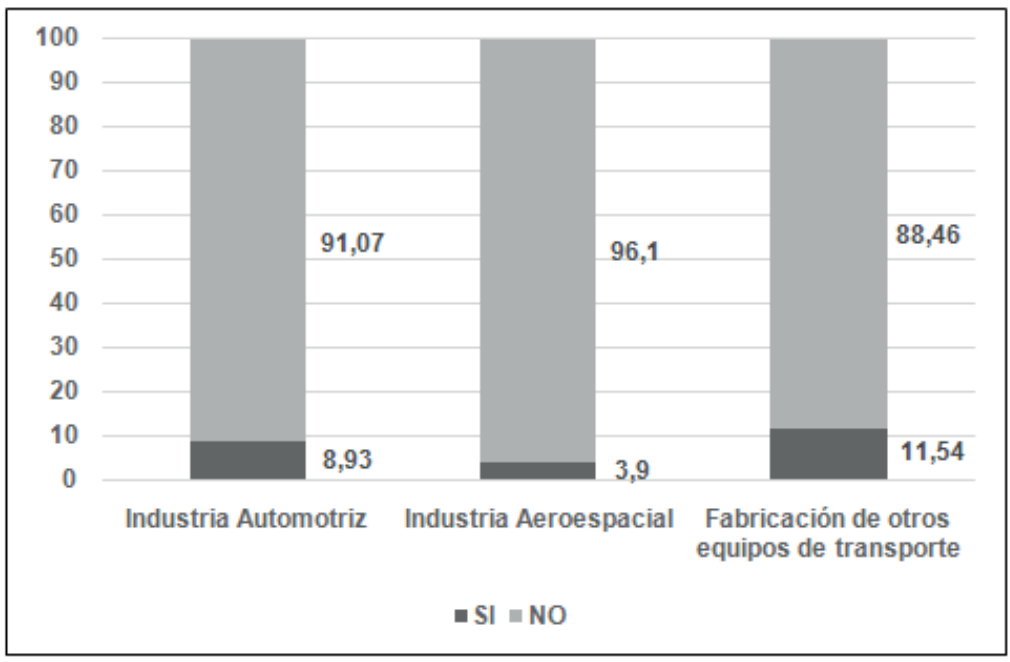

Fuente: elaboración propia con base en INEGI (2014)

El gráficco 2, muestra que existe un porcentaje mayor de participación de las empresas dentro de cada rama en lo que respecta al gasto o inversión para reducir la contaminación en aguas residuales generadas, en contaposición, son las compañías aeroespaciales las que muestran una contribución relativa más alta en el grupo de su rama de actividad. Este hallazgo del bajo número de empresas manufactureras que gastan o invierten para reducir la contaminación que producen al aire y agua, es muy parecido al de González et al, (2007a:16), quienes encontraron que en México sólo 58 unidades productivas de una muestra de 10200 gastaron en investigación y desarrollo tecnológico con el propósito de control y protección del medio ambiente. 


\section{Gráfico 2 \\ Gasto corriente o de inversión para disminuir los contaminantes en aguas residuales generadas}

(\%)

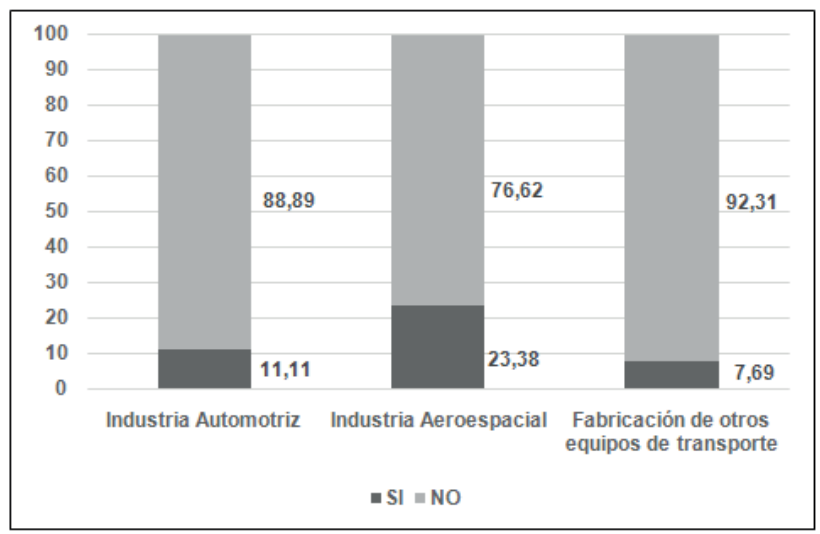

Fuente: elaboración propia con base en INEGI (2014)

Un poco más de la mitad de las unidades económicas aglutinadas en el grupo de la industria automotriz respondieron que cuentan con personal dedicado a la protección del medio ambiente y los recursos naturales, en contraste, apenas un poco por arriba de un cuarto de los establecimientos de la industria aeroespacial manifestaron que tienen este tipo de personal especializado (Gráfico 3).

Un resultado muy parecido fue el que obtuvo Muller y Kolk (2009:330), quienes enconraron que aproximadamente tres cuartas partes de las empresas de autopartes que encuestaron (193) dedican alrededor de media día a capacitar trabajadores no administrativos en aspectos de mejora al medio ambiente, tales como la disminución de desechos industriales, uso eficiente de la energía o el cuidado del agua

Uno de los pilares de la regulación ambiental tiene que ver con las normas ambientales, en este aspecto alrededor de la mitad de las unidades económicas de las ramas automotriz y aeroespacial cumplieron con alguna norma ambiental (Gáfico 4), no obstante, obsérvese que es considerable la cantidad de empresas del subsector que no obedecen o desconocen cumplir con alguna normatividad de carácter ambiental. Este dato sugiere que las medidas de mandato y control son muy limitadas para involucrar a toda una industria o actividad económica a mejorar su desempeño ambiental. 


\section{Gráfico 3}

Empleados dedicados a ejecutar acciones de protección del medio ambiente o de los recursos naturales (\%)

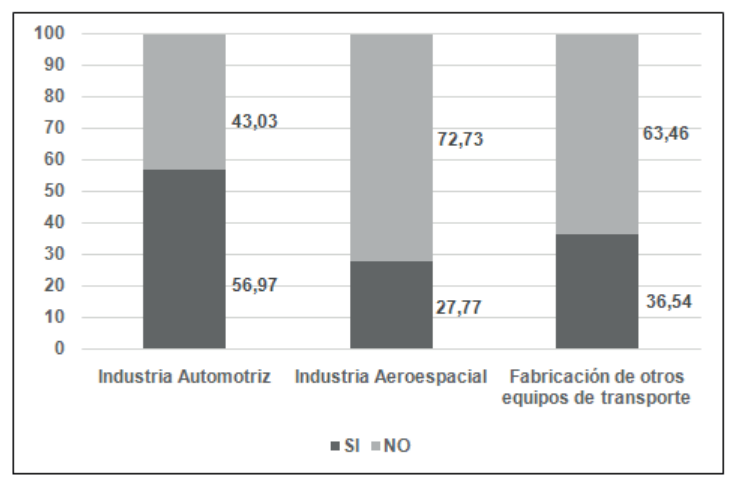

Fuente: elaboración propia con base en INEGI (2014)

\section{Gráfico 4 \\ Cumplimiento de normas relacionadas con el medio ambiente (\%)}

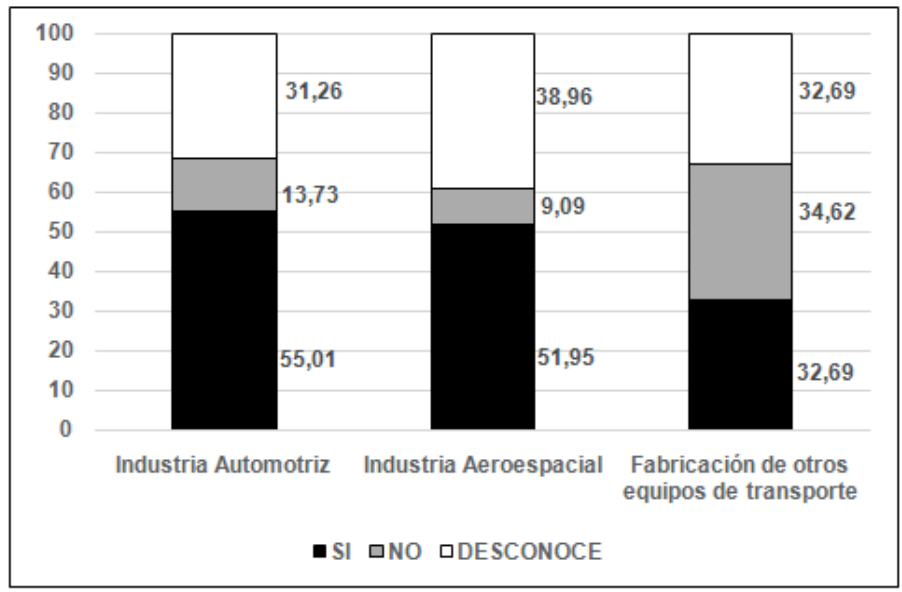

Fuente: elaboración propia con base en INEGI (2014) 
Prácticas ambientales en empresas manufactureras de equipo de transporte en México González Acolt, Roberto; Castillo Rodríguez, Jesús; Ivanova Boncheva, Antonina

En los gráficos 5 y 6 , se puede observar la práctica ambiental de estas empresas con relación a los materiales reciclados, se nota que el empleo de materias primas recicladas es muy bajo dentro de las unidades económicas de la rama aeroespacial, en esta práctica, las industrias de la rama automotriz muestran un mejor desenvolvimiento, no obstante, se puede considerar que es bajo el reciclaje de materias primas en las empresas dentro de las tres ramas en que se agrupó el subsector. Una participación más significativa de estas unidades productivas se nota en el uso del reciclaje de materiales de empaque y embalage. Si se considera en conjunto estas dos formas de reciclaje, las unidades económicas del subsector de equipo de transporte muestran un patrón similar al que detectaron Aigner y Lloret (2013:1258), quienes basados en una muestra de 103 empresas hallaron que alrededor del $39 \%$ de ellas recuperan los desechos que generan

\section{Gráfico 5}

Materiales reciclados (materias primas) en la actividad manufacturera (\%)

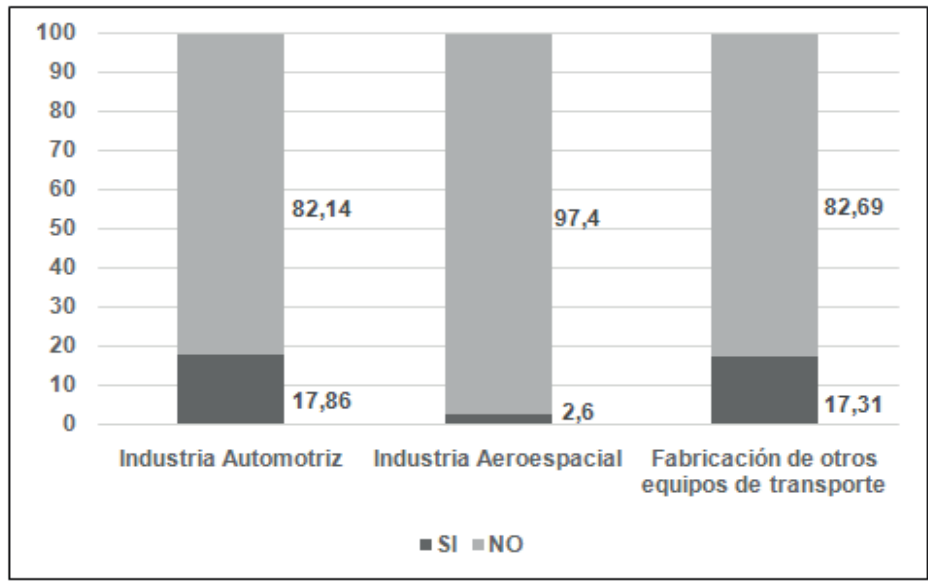

Fuente: elaboración propia con base en INEGI (2014) 


\section{Gráfico 6 \\ Materiales reciclados (empaque y embalaje) en la actividad fabricación de equipo de transporte (\%)}

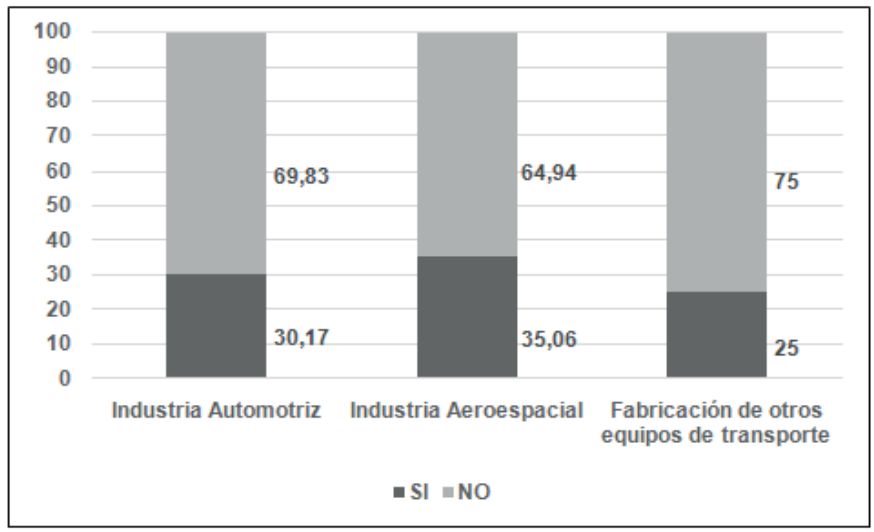

Fuente: elaboración propia con base en INEGI (2014)

Una de las prácticas ambientales donde existe un mayor involucramiento de las unidades económicas de esta actividad manufacturera es la separación de residuos o desechos generados, por ejemplo, aproximadamente el
$88 \%$ de los establecimientos del grupo automotriz aplicarón esta práctica. El grado de participación dentro del grupo de las empresas aeroespaciales fue el más bajo en el subsector de fabricación de equipo de transporte (gráfico 7).

\section{Gráfico 7}

Separación de residuos o desechos generados (\%)

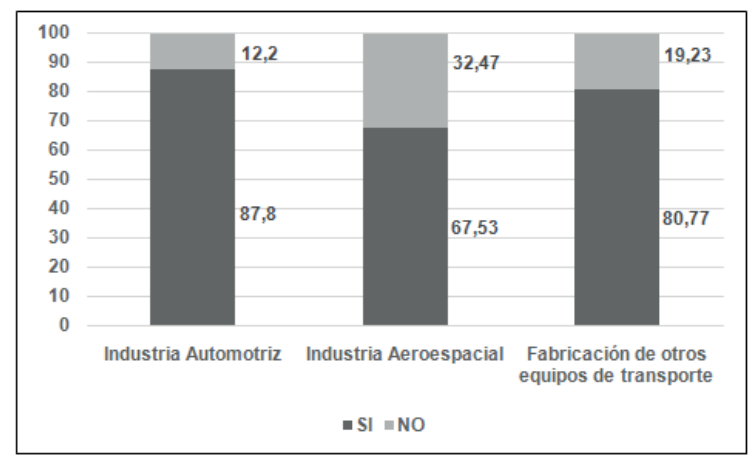

Fuente: elaboración propia con base en INEGI (2014) 
Prácticas ambientales en empresas manufactureras de equipo de transporte en México González Acolt, Roberto; Castillo Rodríguez, Jesús; Ivanova Boncheva, Antonina

El gráfico 8 describe la contribución relativa de las unidades económicas del subsector fabricación de equipo de transporte en la práctica ambiental relacionada con la aplicación de algún tratamiento a las aguas residuales generadas. En este caso son las unidades económicas de la rama aeroespacial -en contraste con los establecimientos de las ramas automotriz y fabricación de otros equipos de transporte-, las que más participaron en la aplicación de esta medida (39\%). Conducta análoga se observa en el trabajo de González et. al (2017b: 164), quienes con el empleo de una encuesta del Banco Mundial a 922 empresas manufactureras, localizarón que alrededor del $50 \%$ de éstas aplicaron programas de optimización del agua.

\section{Gráfico 8}

\section{Aplicación de tratamiento a las aguas residuales (\%)}

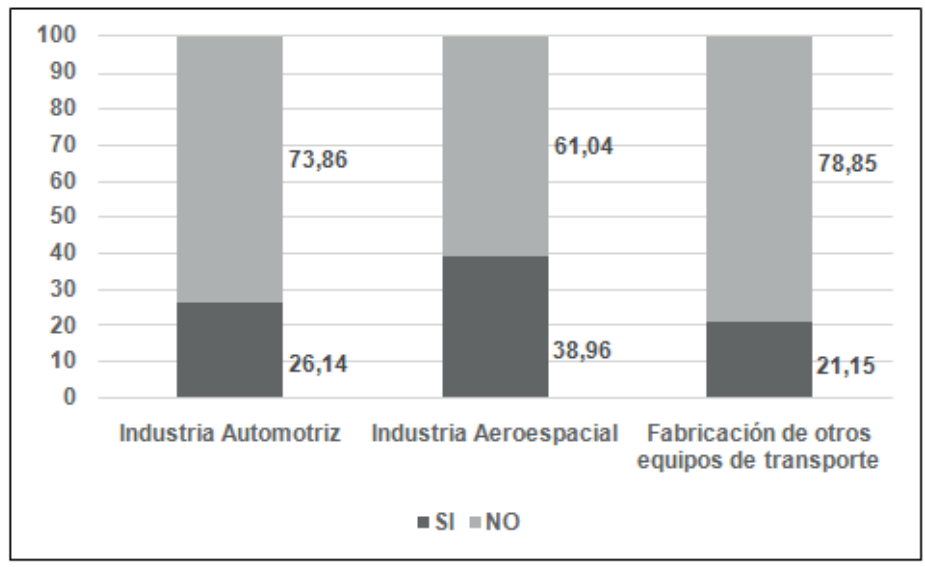

Fuente: elaboración propia con base en INEGI (2014)

De los resultados derivados se desprende que el grado de involucramiento de las empresas del subsector fabricación de equipo de transporte en prácticas de mejoramiento del medio ambiente es heterogéneo, por un lado es muy bajo el número de unidades económicas que gastaron o invirtieron en disminuir la contaminación que generan al aire y agua, en contraposición, una buena parte de estas empresas separa desechos y residuos, con destacada contribución de las firmas de la rama automotriz. Ahora bien, una parte de estos desechos o residuos se pueden volver a reutilizar en el proceso productivo, y tal como se nota en las figuras 5 y 6 las unidades productivas automotrices se destacan por su participación relativa en esta práctica. 


\section{Conclusiones}

En este trabajo se analizó las prácticas ambientales de las empresas del subsector fabricación de equipo de transporte en México, basado en una muestra de 1047 unidades productivas de esta actividad económica. Los datos muestranque elempleo de estas prácticas no esta generalizo en las unidades productivas. En la práctica donde existe un mayor número de empresas participantes es en la separación de residuos y desechos, probablemente, la explicación se centré en el ahorro que implica para las empresas. Una parte de los residuos o desechos separados se recicla, por lo mismo, en esta práctica del reciclaje de materias primas, empaque y embalaje se observa un relativo número de participación de estas unidades productivas en la actividad del reciclaje. Otra parte de los residuos o desechos que se separan se pueden destinar a los rellenos sanitarios o basureros, en este caso la autoridad ambiental debe tener una intervención importante para evitar que se genere una mayor contaminación en el manejo inadecuado de estos materiales.

Por otro lado, todavía es muy escaso el número de unidades productivas del subsector fabricación de equipo de transporte que gastan o invierten en disminuir la contaminación que generan, lamentablemente como lo demuestran otros estudios, esta situación se presenta en empresas ubicadas en otras actividades manufactureras. Esto puede significar que todavía es muy lenta la reconversión de los procesos productivos contaminantes por los procesos ambientalmente más limpios de gran parte de las empresas del subsector.

Que el proceso de reconversión o innovación ambiental llama la atención, en la descripción de los resultados, que un poco más de la mitad de las empresas de las ramas automotriz y aeroespacial manifiesten que si cumplen con alguna norma de carácter ambiental. Generalmente, la sociedad tiende a inclinarse por instrumentos normativos para detener o disminuir la contaminación que generan las industrias, no obstante, con los hallazgos de este trabajo se demuestra que el empleo de esta práctica no esta generalizada en una actividad económica importante como es la fabricación de equipo de transporte.

\section{Referencias bibliográficas}

Aigner, Dennis y Lloret, Antonio (2013), Sustainability and competitiveness in Mexico. Management Research Review, vol 36, No. 12, Emerald, pp 1252.1271.

Azqueta, Diego; Alviar, Mauricio; Domínguez, Lilia y O’Rayan, Raúl (2007), Introducción a la Economía Ambiental. Madrid, España: McGraw-Hill.

Brown, Flor (2008), Apertura y respuesta ambiental: la industria textil mexicana. Jenkins, Rhys y Mercado, Alfonso. (Eds), Ambiente e Industria en México: Tendencias, regulación y comportamiento empresarial, Mexico D.F., Colegio de México, pp 135-161.

Earnhart, Dietrich; Khanna, Madhu y Lyon, Thomas (2014), Corporate Environmental Strategies in Emerging Economies. Review of Environmental Economics and Policy, vol. 8, No. 2, Oxford University Press, pp 164-185.

Gonzalez, Roberto; Uscanga, Carolina y Leal, Felipe (2017a), Actividades de innovación ambiental en las 
Prácticas ambientales en empresas manufactureras de equipo de transporte en México González Acolt, Roberto; Castillo Rodríguez, Jesús; Ivanova Boncheva, Antonina

empresas de México. Revista Global de Negocios, vol. 5, No. 3, USA, The Institute for Business and Finance Research, pp. 13-22.

Gonzalez, Roberto; Martínez, Fernando A y Leal, Felipe (2017b), Aspectos ambientales en la RSC de las empresas manufactureras en México. Denarius. Revista de Economía y Administración, No. 32, México,Universidad Autónoma Metropolitana, pp. 153-172.

Hernández, Juana (2011), Transferencia de conocimiento en la industria aeroespacial mexicana: el caso de Bombardier Aerospacial, Queretaro, Revista de Economía del Caribe, No. 7, Colombia, Universidad del Norte, pp 231-269.

Instituto Nacional de Estadística y Geografía INEGI (2014), Censos Económicos 2014, Módulo de medio Ambiente 2014, microdatos, México.

Instituto Nacional de Estadística y Geografía INEGI (2013), Sistema de clasificación industrial de América del Norte, México SCIAN 2013, México.

Instituto Nacional de Estadística y Geografía INEGI (2012), Sistema de Cuentas Nacionales de México. Cuentas económicas y ecológicas de México, 2006-2010, México.

Medina, Veronica (2008), La gestión ambiental voluntaria en el sector químico en México. Jenkins, Rhys y Mercado, Alfonso. (Eds), Ambiente e Industria en México: Tendencias, regulación y comportamiento empresarial, Mexico D.F., Colegio de México, pp 197-246.

Muller, Alan y Kolk, Ans (2009), CSR Performance in emerging markets evidence from Mexico. Journal of Business Ethics, vol 85, No. 2, Springer Netherlands, pp 325-337.

Romero, Indira (2011), Impacto asimétrico de la crisis global sobre la industria automotriz: Canadá y México comparados. Perspectivas para el futuro. México, CEPAL.

Romo, David; Romero, Omar y Samaniego, Ricardo (2005), Industria y medio ambiente en México: Hacia un nuevo paradigma para el control de la contaminación. México, D.F., H. Cámara de Diputados, LIX Legislatura; ITAM; Miguel Ángel Porrúa.

Unger, Kurt (2010), Globalización y clusters regionales en México: Un enfoque evolutivo, Mexico D.F.: Fondo de Cultura Económica.

- Esta obra está bajo una licencia de Creative Commons Reconocimiento-NoComercialCompartirlgual 3.0 Unported. http://creativecommons.org/licenses/by-nc-sa/3.0/deed.es_ES 